\title{
The Un-managed System of Medicare Referrals
}

\author{
Alice $\mathrm{Hm}$ Chen, $\mathrm{MD}, \mathrm{MPH}^{7}$ and Diane Rittenhouse, $\mathrm{MD}, \mathrm{MPH}^{2}$ \\ 'Division of General Internal Medicine, Department of Medicine \& Center for Innovation in Access and Quality, University of California, \\ San Francisco at San Francisco General Hospital, San Francisco, CA, USA; ${ }^{2}$ Department of Family and Community Medicine \& Philip R. Lee \\ Institute for Health Policy Studies, University of California, San Francisco, San Francisco, CA, USA.
}

J Gen Intern Med 27(5):487-9

DOI: $10.1007 / \mathrm{s} 11606-012-2014-8$

(C) Society of General Internal Medicine 2012

$\mathrm{S}$ ince the passage of the Affordable Care Act two years ago, patient centered medical homes (PCMH) and accountable care organizations (ACO) have emerged as leading models to address our fragmented, high cost health care system. ${ }^{1}$ The Center for Medicare and Medicaid Innovation (CMMI) has been allocated $\$ 10$ billion over a decade to test and spread these and other new models of care and payment, with the aim of providing better health for individuals and populations at lower cost.

Last December, CMMI chose 32 organizations across the country to participate in its "Pioneer ACO" program. These ACOs are defined as "groups of doctors, hospitals, and other health care providers, who come together voluntarily to give coordinated high quality care to the Medicare patients they serve." 2 If the ACOs provide care for their patients at a lower cost than would otherwise be predicted, they share in the cost savings; if their costs are higher than predicted, they share the losses.

Notable elements of the program include the preservation of consumer choice and the lack of any requirement for beneficiaries to have a primary care provider or medical home. CMMI emphasizes that patients have complete freedom to visit any healthcare provider accepting Medicare at any time, "just as all Medicare beneficiaries participating in original, fee-for-service Medicare do."

Indeed, Medicare has been called the "lone bastion of largely unmanaged fee-for-service care in the United States."3 Many Medicare patients do not have a connection with a primary care physician (PCP) at all: in one analysis, $24 \%$ of patients had a specialist physician as their usual source of care; in another, $14 \%$ of patients saw only a specialist over the course of a year. ${ }^{3,4}$ For those who do have a PCP, the coordination challenges are mind-boggling: for every 100 Medicare beneficiaries, the average PCP needs to coordinate with 99 physicians in 53 different practices. ${ }^{5}$

Published online February 29, 2012
In this issue of JGIM, Barnett and colleagues report the results of a survey whose findings highlight some of the challenges in providing coordinated, patient-centered care for Medicare beneficiaries in the current model. ${ }^{6}$ The authors used administrative data for PCPs, medical subspecialists, and surgical subspecialists to identify physician colleagues based on shared Medicare patients. Participating physicians were presented with their professional network of Medicare colleagues, and were asked to identify individuals from this list to whom they referred. They were also asked to provide up to two reasons - aside from clinical expertise-why they referred to that particular physician. While the study is limited by its geographic focus and academic setting, in the context of health reform and attempts to foster delivery system integration and coordination of care, three findings deserve discussion.

First, medical and surgical subspecialists reported initiating referrals to approximately half (49\% and 52\%, respectively) of the physicians within their identified professional network of Medicare colleagues, as compared with $66 \%$ for PCPs. For both medical and surgical subspecialists, a distinct minority of the referral relationships were with PCPs (10\% and $20 \%$ respectively). About a third of their referral relationships were with surgical subspecialists, while the majority were with medical subspecialists.

The issue of specialist-to-specialist referrals, variously referred to as "cross-referrals" or "secondary referrals," is not well characterized in the literature. In one study of nonMedicare patients, the rate was low, approximately $3 \%{ }^{7}$ However, in a prior analysis of Medicare referrals, researchers found a multi-directional pattern consistent with the findings in this study, with sizable numbers of referrals from PCPs to PCPs, specialists to PCPs, and specialists to specialists. ${ }^{3}$

From a primary care perspective, the high prevalence of secondary referral relationships among specialists is concerning. Although some likely represent specialists acting on behalf of their patients in the absence of a defined primary care relationship, surely many secondary referrals bypass an established primary care home whose charge is to provide first contact, continuous, coordinated, comprehensive care. High rates of secondary referrals suggest that patients may not be receiving the benefits of primary care services, and are at greater risk for disjointed, duplicative care. 
While there are clinical situations where a secondary referral may be appropriate for streamlining and expediting care, for example from a general cardiologist to an electrophysiologist or from an endocrinologist to a pituitary surgeon, to ensure that care is integrated from the patient's perspective, all cases should include communication and possibly consultation with the primary care home. Underscoring this point, the American College of Physicians has developed a patientcentered medical neighborhood model that recommends care coordination agreements between primary care and specialist physicians that explicitly address how both secondary referrals and patient self-referrals should be handled. ${ }^{8}$

The second finding of note is that PCPs, medical subspecialists and surgical subspecialists differed in the reasons they provided for choice of referral colleague. Compared with medical subspecialists, PCPs were significantly more likely to be concerned with between-physician communication (a composite measure), and compared with surgical subspecialists, they placed significantly greater import on whether a colleague shared their medical record (18\% versus less than $3 \%)$.

This finding is congruent with the different roles played by primary care and subspecialist physicians. While subspecialists provide deep but narrow expertise in a particular organ system or disease, the role of PCPs is to provide a much broader longitudinal perspective, focusing on the whole patient over time. Excellent flow of information enables PCPs to coordinate across body systems, health care providers and settings, and to assist patients in setting goals and priorities. At the same time, given the sheer number of physicians and practices involved in a typical Medicare patient's care, the responsibility for communication cannot lie entirely with PCPs. A key attribute of a functioning medical neighborhood is proactive communication by subspecialists. ${ }^{8}$ Particularly in the context of self-referrals and secondary referrals, ACOs will need to create processes and systems to ensure ready access to timely clinical information that spans the care continuum.

The last salient finding is that all three groups were unlikely to cite "patient access" factors as being considerations in choosing a colleague for referral: roughly a third of PCPs, a quarter of medical subspecialists and a fifth of surgical subspecialists chose this category. Patient access factors such as timely availability of appointments, convenient location, language concordance, or patient request were arguably the most explicitly patient-centered measures in the survey.

The concept of patient-centered care was first introduced by the Institute of Medicine more than a decade ago. Since then, this verbiage has been incorporated into many key policy initiatives, including patient centered medical homes and the Patient Centered Outcomes Research Institute, but has largely remained aspirational in nature. A 2004 survey of American adults in primary care practices found that $44 \%$ of respondents reported that their physicians sometimes, rarely or never told them treatment choices or asked for their opinion in making decisions about their care. ${ }^{9}$ More recently, a 2009 survey found that among patients referred to a specialist, $26 \%$ reported feeling unprepared about what to expect, and a similar percentage received no information regarding follow-up from the specialist. ${ }^{10}$ We still have a long way to go to transform our provider-driven system into one that places patients at its center.

As health reform calls for the reorganization of health care into integrated systems that are accountable for patient outcomes and total spending, the survey findings from Barnett and colleagues highlight several important challenges: a high prevalence of secondary referral relationships; a difference in orientation between PCPs and subspecialists in valuing communication and shared clinical information; and relatively low priority placed on patient factors when choosing a referral colleague.

While physicians' reasons for referral may be generalizable, the extent of secondary referrals is likely driven by the study's focus on Medicare, which arguably represents a worst case scenario for integration. Most Americans are currently in managed care arrangements that, to a greater or lesser extent, either incentivize against or ban self-referrals and limit physician networks. Traditionally these networks have been primarily driven by cost considerations, but more recently insurers have begun designing high performance networks based on quality and cost data. ${ }^{11}$ There has also been interest in leveraging information technology to more easily incorporate patient and clinician preferences into the referral selection process. ${ }^{12}$

Future studies should better define the ecology of secondary referrals, including referral rates, patterns of subspecialty referral, indications for referral, involvement of PCPs, patient safety considerations, and cost implications. It will be important to track how the recent $\$ 29$ billion federal investment in health information technology, adoption of electronic health records and development of health information exchanges shapes referral decisions and networks over time. And much more attention needs to be paid to patient experience and patient preferences in the referral process.

Meanwhile, the Pioneer ACO program is being presented to patients as a way to improve their healthcare experience:

Any patient who has multiple doctors probably understands the frustration of fragmented and disconnected care: lost or unavailable medical charts, doctors who don't coordinate their decisions with one another, duplicated medical procedures, or having to share the same information over and over with different doctors. Accountable care organizations are designed to lift this burden from patients, while improving the partnership between patients and doctors in making health care decisions. ${ }^{2}$ 
Whether or not ACOs deliver on this promise will depend on whether they are able to realign incentives, relationships and expectations among PCPs, subspecialists and patients in the service of less fragmented and more efficient care.

Corresponding Author: Alice Hm Chen, MD, MPH; Division of General Internal Medicine, Department of Medicine \& Center for Innovation in Access and Quality, University of California, San Francisco at San Francisco General Hospital, Box 1364, San Francisco, CA 94143-1364, USA (e-mail: achen@medsfgh.ucsf.edu).

\section{REFERENCES}

1. Rittenhouse DR, Shortell SM, Fisher ES. Primary care and accountable care - two essential elements of delivery-system reform. N Engl J Med. 2009;361:2301-2303.

2. Centers for Medicare \& Medicaid Services. Pioneer Accountable Care Organization (ACO) Model Program Frequently Asked Questions. Available at: http://innovations.cms.gov/documents/pdf/PioneerACO-external FAQs_FINAL_12_20_11.pdf. Accessed January 9, 2012.
3. Shea D, Stuart B, Vasey J, Nag S. Medicare physician referral patterns. Health Serv Res. 1999;34(1 Pt 2):331-348.

4. Xakellis GC. Are patients who use a generalist physician healthier than those who seek specialty care directly? Fam Med. 2005;37(10):719-726.

5. Pham HH, O'Malley AS, Bach PB, Saiontz-Martinez C, Schrag D. Primary care physicians' links to other physicians through Medicare patients: the scope of care coordination. Ann Intern Med. 2009;150 (4):236-242.

6. Barnett ML, Keating NL, Christakis NA, O'Malley AJ, Landon BE. Reasons for choice of referral physician among primary care and specialist physicians. J Gen Intern Med. 2011; doi:10.1007/s11606-011-1861-z.

7. Forrest CB, Reid RJ. Passing the baton: HMOs' influence on referrals to specialty care. Health Aff (Millwood). 1997;16(6):157-162.

8. Laine C. Welcome to the patient-centered medical neighborhood. Ann Intern Med. 2011;154(1):60.

9. Schoen C, Osborn R, Huynh PT, Doty M, Davis K, Zapert K, et al. Primary care and health system performance: adults' experiences in five countries. Health Aff (Millwood). 2004 Jul-Dec; Suppl Web Exclusives: W4-487-503.

10. Ireson CL, Slavova S, Steltenkamp CL, Scutchfield FD. Bridging the care continuum: patient information needs for specialist referrals. BMC Health Serv Res. 2009;9:163.

11. Pham HH, Ginsburg PB, McKenzie K, Milstein A. Redesigning care delivery in response to a high-performance network: the Virginia Mason Medical Center. Health Aff (Millwood). 2007;26(4):w532-w544.

12. Einbinder JS, Klein DA, Safran CS. Making effective referrals: a knowledge-management approach. Proc AMIA Annu Fall Symp. 1997;330-4. 